\title{
MộT SỐ TÁC ĐộNG CỦA BÀI THI ĐÁNH GIÁ NĂNG LỰC TIẾNG ANH THEO CHUẨN ĐÂU RA ĐỐI VỚI VIỆC DẠY TIẾNG ANH TẠI TRƯÒ̀ ĐẠI HỌC NGOẠI NGŨ் - ĐẠI HỌC QUỐC GIA HÀ NỘI
}

\author{
Nguyễn Thúy Lan* \\ Phòng Đào tạo, Truò̀ng Đại học Ngoại ngũu, ĐHQGHN, \\ Phạm Văn Đồng, Cầu Giấy, Hà Nội, Việt Nam \\ Nhận bài ngày 16 tháng 02 năm 2017 \\ Chỉnh sửa ngày 28 tháng 06 năm 2017; Chấp nhận đăng ngày 26 tháng 07 năm 2017
}

Tóm tắt: Từ lâu, kiểm tra đánh giá kết quả học tập và quá trình dạy học đã có mối quan hệ biện chứng chặt chẽ. Kiểm tra đánh giá (KTĐG) khi xuất hiện ở khâu cuối của quá trình đào tạo sẽ đưa ra được thông tin về mức độ hoàn thành mục tiêu đào tạo của người học; tuy nhiên, nó còn có tác dụng điều tiết trở lại hết sức mạnh mẽ đối với quá trình đào tạo. Qua gần 2 năm được sử dụng làm bài thi chính thức xác định chuẩn đầu ra năng lực tiếng Anh tại Trường Đại học Ngoại ngữ - Đại học Quốc gia Hà Nội (ĐHNN - ĐHQGHN), bài thi VSTEP đã bước đầu có những tác động lớn tới quá trình dạy và quản lý đào tạo các học phần thực hành tiếng (ngôn ngữ Anh) của nhà trường. Bài viết này bước đầu phát hiện ra một số tác động tích cực và một số tác động cần sự chú ý của các nhà quản lý, qua đó có một số đề xuất nhằm nâng cao hiệu quả của bài thi trong việc định hướng lại cho quá trình đào tạo của nhà trường.

Tù khóa: tác động, đánh giá năng lực tiếng Anh, chuẩn đầu ra

\section{1. Đặt vấn đề}

"Đề án dạy và học ngoại ngữ trong hệ thống giáo dục quốc dân giai đoạn 20082020" đã có những chương trình hành động quyết liệt trong lộ trình đổi mới toàn diện việc dạy và học ngoại ngữ của quốc gia. Dựa trên Khung năng lực ngoại ngữ 6 bậc dành cho Việt Nam (KNLNN) - một sản phẩm của đề án, yêu cầu chuẩn đầu ra về ngoại ngữ đối với các cấp học sau phổ thông được qui định bậc 3 đối với khối không chuyên, bậc 4 đối với khối chuyên ngoại ngữ bậc cao đẳng, bậc 5 đối với khối chuyên ngoại ngữ bậc đại học. Tiếp theo đó, ngày 11/3/2015, Bộ Giáo dục và Đào tạo đã ban hành quyết định 729/QĐ-BGDĐT chính thức công nhận Đề thi đánh giá năng lực sử dụng tiếng Anh từ bậc 3 đến bậc 5 theo KNLNN (VSTEP) là một công cụ đánh giá năng lực tiếng Anh từ bậc $3-5$ cho đối tượng

\footnotetext{
*ĐT.: 84-928003530

Email: lanthuy.nguyen@gmail.com lannt.ulis@vnu.edu.vn
}

sau trung học phổ thông, được sử dụng trong toàn hệ thống giáo dục quốc dân và hướng tới được quốc tế công nhận.

Sau khi được ban hành, VSTEP chính thức trở thành bài thi tiếng $\mathrm{Anh}$ nhằm xác định chuẩn đầu ra ngoại ngữ cho tất cả sinh viên các trường thuộc Đại học Quốc gia Hà Nội (ĐHQGHN), trong đó có Trường Đại học Ngoại ngữ (ĐHNN). Sinh viên khối chuyên của ĐHNN cần đạt được bậc 5/6 mới đủ điều kiện xét tốt nghiệp và đây là một áp lực không nhỏ đối với cả người học, người dạy và các nhà quản lý.

Trong khuôn khổ bài viết này, chúng tôi bước đầu muốn phát hiện và phân tích một số tác động của bài thi VSTEP tới quá trình dạy và quản lý đào tạo các học phần thực hành tiếng thuộc chương trình đào tạo Ngôn ngữ Anh/Sư phạm Anh của ĐHNN - ĐHQGHN. 
2. Tác động của KTĐG đối với hoạt động dạy ngoại ngũ̃

2.1. Lịch sử nghiên cứu tác động dội ngược (washback effects) của KTĐG đối vói hoạt động dạy ngoại ngũ

Lịch sử nghiên cứu tác động dội ngược của các bài kiểm tra đối với hoạt động dạy và học được bắt đầu từ những năm 1950 và 1960. Các nghiên cứu đều chỉ ra rằng các bài kiểm tra có ảnh hưởng mạnh mẽ đến việc lựa chọn tài liệu và thiết kế hoạt động giảng dạy của giáo viên. Theo Vernon (1956), giáo viên thường không chú ý đến những kiến thức không trực tiếp liên quan đến các bài kiểm tra, do đó các mục tiêu của chương trình đào tạo thường không được quan tâm đầy đủ. Trong một nghiên cứu khác, Davies (1968) cũng chỉ ra rằng giáo viên thường sử dụng các bài kiểm tra và tài liệu ôn tập cho các kỳ thi làm tài liệu dạy học. Việc lựa chọn tài liệu này làm thu hẹp những trải nghiệm giáo dục của người học. Popham (1983) là người đầu tiên đưa ra khái niệm dạy học theo hướng đánh giá đo lường (measurement-driven instruction). Khái niệm này nhấn mạnh việc thống nhất giữa dạng thức, nội dung của bài kiểm tra với cấu trúc và nội dung chương trình học. Cũng đã có nghiên cứu gần đây hơn chỉ ra rằng khi đưa ra một bài kiểm tra mới vào một bối cảnh giáo dục cụ thể có thể mang lại những hiệu quả tích cực đối với việc dạy và học. Đó là những khái niệm như tính giá trị hệ thống (systematic validity), tính giá trị hệ quả (consequential validity) hay ảnh hưởng của bài kiểm tra (test impact).

\section{2. Định nghĩa tác động dộingược (washback effects) trong $K T Ð G$}

Các nghiên cứu trong lĩnh vực kiểm tra đánh giá đều khẳng định các bài thi, đặc biệt là bài thi có tỉ suất đánh giá cao (highstakes test) có ảnh hưởng lớn đến hoạt động dạy và học. Những ảnh hưởng này thường được gọi là các "tác động dội ngược" (washback effects). Khái niệm này đã được định nghĩa theo nhiều cách khác nhau trong lịch sử nghiên cứu vấn đề.

Alderson \& Wall (1993) và Biggs (1995, 1996) định nghĩa "tác động dội ngược" (washback hay backwash) là ảnh hưởng của bài thi ngược trở lại với quá trình dạy và học. Khái niệm này xuất phát từ quan điểm cho rằng $\mathrm{KT} Đ \mathrm{G}$ có thể và nên định hướng cho quá trình dạy và học. Theo Alderson \& Wall (1993), tác động dội ngược chỉ đề cập đến hành vi của người học và giáo viên trong khuôn khổ lớp học khi chịu ảnh hưởng của một bài thi cụ thể.

Để làm rõ mức độ và phạm vi tác động của bài thi, nhiều tác giả đã phân biệt rõ giữa tác động dội ngược (washback) và ảnh hưởng (impact) của bài thi. Wall (1997) chî ra rằng: "ảnh hưởng của bài thi” là “....bất cú ảnh hưởng nào của bài thi tới cá nhân, chính sách trong lớp học, trường học, hệ thống giáo dục hay toàn xã hội”; trong khi đó tác động dội ngược của bài thi chỉ đề cập đến "những ảnh hưởng của bài thi tới việc dạy và học" (tr. 291). Tương tự, Shohamy (2001) cho rằng tác động dội ngược (washback effects) là một thành tố nằm trong ảnh hưởng của bài thi (test impact). Ảnh hưởng của bài thi diễn ra trên phạm vi xã hội hay một cơ sở giáo dục; tác động dội ngược diễn ra trên phạm vi cá nhân người học và người dạy.

Hiện nay, tác động dội ngược (washback effect) đã được xem là một khía cạnh của độ giá trị của một bài thi, và được gọi bằng một thuật ngữ khá mới mẻ là "giá trị hệ quả" (consequential validity), nhằm nhấn mạnh "hệ quả" của việc thi cử, kiểm tra đánh giá lên việc dạy và học trước đó.

\subsection{Phân loại tác động dội ngược}

Có một số cách phân loại tác động dội ngược của bài thi. Với quan điểm về sự khác biệt rõ ràng giữa ảnh hưởng của bài thi và tác 
động dội ngược của bài thi, Bailey (1996, 1999) chia nhỏ tác động dội ngược thành hai loại: tác động tới người học và tác động tới chương trình. Tác động tới người học đề cập đến những ảnh hưởng của bài thi tới người học; tác động tới chương trình đề cập đến ảnh hưởng đối với những người khác như giáo viên, người phát triển tài liệu và các nhà quản lý.

Tuy nhiên, cách phân loại phổ biến nhất vẫn dựa trên chất lượng của việc thiết kế bài thi. Hwang (2003) cho rằng: "Nếu bài thi có chất lượng tốt, nó sẽ có tác dụng hỗ trợ việc học, nhưng nếu bài thi có chất lượng tồi, nó sẽ có tác dụng tiêu cực tới việc học" (tr. 16). Những chuyên gia trong lĩnh vực KTĐG đều coi tác động dội ngược có thể tích cực, tiêu cực hay trung tính, tạo ra những hệ quả có thể hoặc không thể lường trước. Một số chuyên gia còn coi một bài thi với các tác động dội ngược của nó có thể là một công cụ tiềm năng tạo ra những cải cách giáo dục.

\subsubsection{Tác động dội ngược tiêu cực}

Có khá nhiều nghiên cứu khẳng định tác động dội ngược tiêu cực của KTĐG. Alderson \& Wall (1993) định nghĩa tác động dội ngược tiêu cực là những ảnh hưởng không mong muốn của một bài thi đối với việc dạy và học. Theo Shohamy (2001), tác động dội ngược của một bài thi là tiêu cực nếu "chương trình luyện thi làm giảm thời gian dạy học, thu hẹp chương trình và phương pháp giảng dạy, có thể làm giảm năng lực dạy nội dung và năng lực sử dụng phương pháp, tài liệu phù hợp định dạng bài thi theo tiêu chuẩn của giáo viên" (tr. 18). Tsagari (2011) phát hiện ra rằng bài thi sẽ có tác động tiêu cực tới việc dạy và học nếu bài thi khiến người dạy và người học tập trung quá nhiều vào nội dung và kỹ năng được kiểm tra mà coi nhẹ những kiến thức và kỹ năng không được kiểm tra. Trong trường hợp này, bài thi đã làm méo mó chương trình học. Damankesh \& Babaii (2015) cho rằng để chuẩn bị cho kỳ thi, người học đã luyện tập bằng cách xem lại các bài thi cũ, tham khảo các sách liên quan đến kỳ thi. Vì lý do đó, chương trình học bị thu hẹp, các hoạt động học tập bị hạn chế và trọng tâm được chuyển từ phát triển năng lực giao tiếp bằng ngôn ngữ sang học thuộc từ vựng, ngữ pháp. Đề cập đến ảnh hưởng về mặt tâm lý, Tsagari (2011) cho rằng bài thi có tác động rất tiêu cực tới thái độ của người học đối với việc học ngôn ngữ do việc chuẩn bị cho bài thi làm họ quá căng thẳng dẫn đến chán nản, không có hứng thú học.

\subsubsection{Tác động dội ngược tích cực}

Alderson \& Wall (1993) định nghĩa tác động dội ngược tích cực là những ảnh hưởng có lợi tới hoạt động dạy và học. Theo Davies (1985), tác động dội ngược của bài thi sẽ là tích cực nếu bài thi đó thúc đẩy được việc dạy và học. Messick (1996) cho rằng "để đạt được hiệu quả tối đa, không nên có sự khác biệt nào giữa hoạt động học ngôn ngữ và hoạt động chuẩn bị cho bài thi"” (tr. 241-242).

\subsection{Các mô hình tác động dội ngược}

Đã có nhiều mô hình lý thuyết về tác động dội ngược được đề xuất; trong đó hai mô hình căn bản và được nhiều nghiên cứu sử dụng nhất là mô hình của Hughes (1993) và Bailey (1996). Mỗi một mô hình có cách tiếp cận vấn đề khác nhau, nhưng đều đề cập đến 3 thành tố chính: người tham gia (participants), quá trình (process) và sản phẩm (product).

Dựa trên khung lý thuyết của Hughes (1993), Bailey (1996) đã bổ sung và đề xuất ra một mô hình lý thuyết về tác động dội ngược cũng gồm 3 thành tố chính: nguoòi tham gia, quá trình và kết quả (xem hình 1). Mối quan hệ giữa 3 thành tố này cũng tương tự như trong mô hình của Hughes: bài thi tác động trực tiếp tới nguời tham gia (người học, người dạy, người viết tài liệu và thiết kế chương trình và nhà nghiên cứu), người tham gia thực hiện quá trình (dạy và học) để đạt được kết quả (những kiến thức, kỹ năng mà người học 
thu nhận được). Điểm khác biệt trong mô hình của Bailey đó là nhà nghiên cứu được coi là một thành tố trong những người tham gia. Về phạm vi tác động, bài thi không chỉ ảnh hưởng hoạt động dạy và học mà còn ảnh hưởng tới những khía cạnh khác như phát triển tài liệu học. Mối quan hệ giữa các thành tố cũng là mối quan hệ biện chứng chứ không phải tuyến tính một chiều. Ví dụ như: bài thi không chỉ làm ảnh hưởng đến kết quả học tập thông qua những người tham gia và quá trình học tập, mà người tham gia và quá trình tham gia của họ cũng đưa thông tin phản hồi lại và ảnh hưởng ngược tới bài thi. Theo Wall (1997), nhược điểm trong mô hình của Bailey là chưa làm rõ được các quá trình trung gian. Nói cách khác, mô hình cho thấy bài thi tác động trực tiếp lên người tham gia mà chưa tính đến vai trò của thái độ, niềm tin sẵn có của người tham gia.
2.5. Một số nghiên cúu liên quan về tác động dội ngược của bài thi tới quá trình dạy ngoại ngũ

Một bài thi có thể ảnh hưởng tới nhiều khía cạnh của quá trình dạy và học. Theo Cheng, Watanabe và Curtis (2004), những yếu tố gây ra ảnh hưởng bao gồm: yếu tố bài thi (phương thức kiểm tra, nội dung kiểm tra, kỹ năng kiểm tra, mục đích kiểm tra, quyết định đưa ra dựa vào kết quả bài thi, v.v.), yếu tố danh tiếng (vị trí của bài thi trong hệ thống giáo dục quốc dân, lợi ích bài thi mang lại cho thí sinh, v.v), yếu tố cá nhân (ví dụ: nền tảng giáo dục của giáo viên, quan niệm của giáo viên về phương pháp dạy và học tốt nhất, v.v), yếu tố bối cảnh tầm vi mô (ví dụ: bối cảnh trường học nơi bài thi được áp dụng), yếu tố bối cảnh tầm vĩ mô (hệ thống xã hội nơi bài thi được áp dụng).

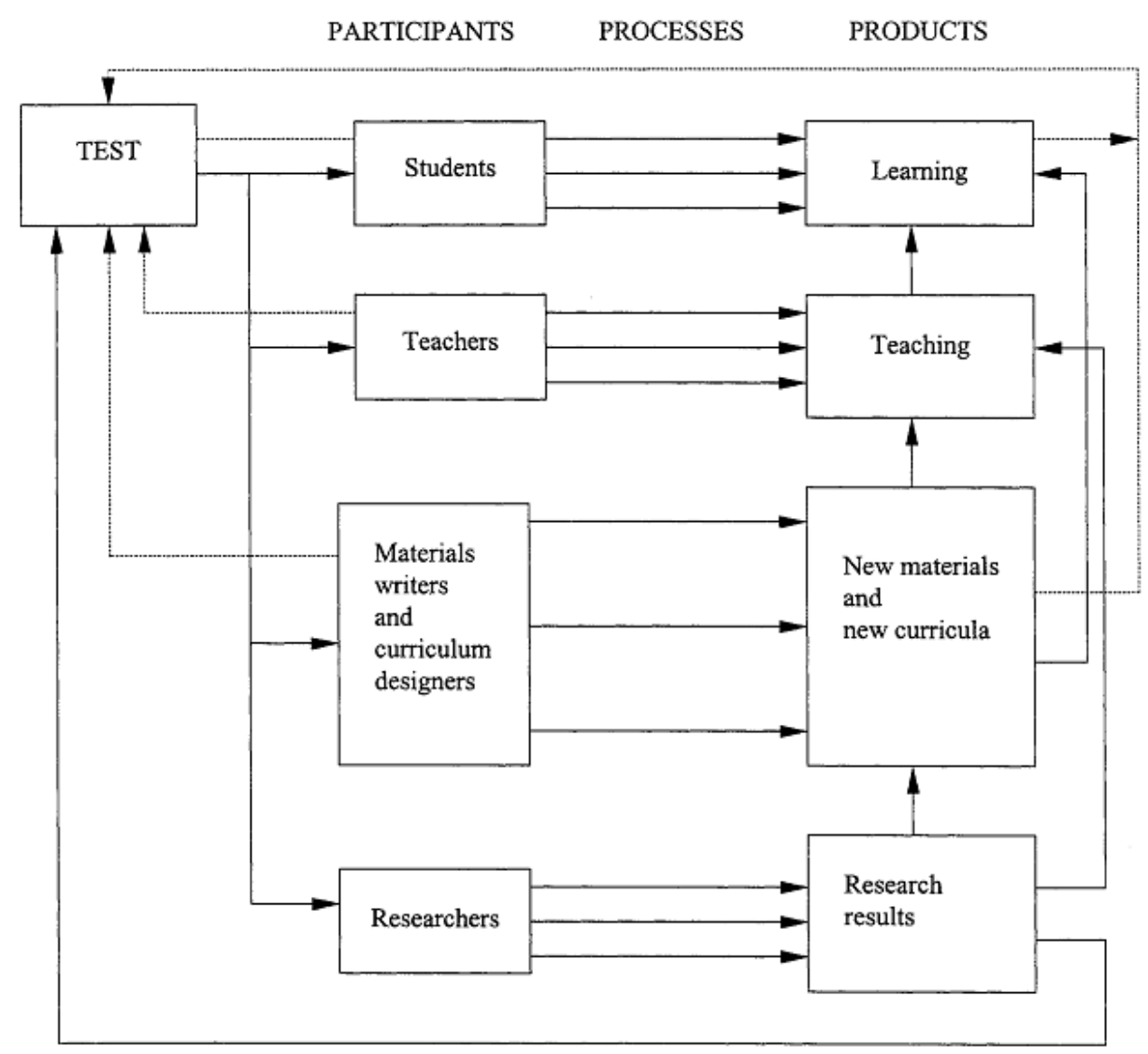

Hình 1. Mô hình tác động dội ngược của Bailey (1996) 
Shohamy (1992) đã tìm hiểu ảnh hưởng của bài thi tiếng Ả rập (ngôn ngữ thứ hai) và tiếng Anh (ngôn ngữ nước ngoài) đối với việc dạy của giáo viên ở Israel. Công cụ thu thập thông tin là bảng hỏi và phỏng vấn. Shohamy và đồng nghiệp đã phát hiện ra rằng khi bài thi tiếng Ả rập được bắt đầu áp dụng, giáo viên không sử dụng tài liệu giảng dạy mới nữa mà bắt đầu ôn tập lại tài liệu cũ; giáo trình được thay thế bởi các tờ bài tập giống như bài thi của những năm trước; các hoạt động trong lớp có định hướng giống bài thi; không khí lớp học trở nên căng thẳng; học sinh và giáo viên có xu hướng cố gắng làm nhuần nhuyễn những bài tập giống các bài thi. Nghiên cứu cũng cho thấy khi kỳ thi kết thúc, các hoạt động dạy và học theo hướng luyện thi cũng kết thúc. Qua phỏng vấn, các nhà nghiên cứu thấy rằng "khi giáo viên nhận ra rằng kết quả thi của học sinh không có tác động cá nhân trực tiếp nào tới họ, giáo viên trở nên thoải mái và đỡ sợ hơn, vì thế ảnh hưởng của bài thi cũng giảm xuống." (tr. 314).

Hwang (2003) nghiên cứu về ảnh hưởng của bài thi đánh giá năng lực (CSAT) đối với việc dạy và học tiếng Anh tại các trường cấp 2 của Hàn Quốc. Bảng câu hỏi điều tra và phỏng vấn được sử dụng để thu thập số liệu từ giáo viên và học sinh. Nhằm tìm ra mối quan hệ giữa bài thi CSAT, chương trình học và giáo trình, số liệu được phân tích dựa trên danh mục các tiêu chí đánh giá giáo trình của Cunningsworth (1995) và danh mục của Bachman và Palmer (1996). Kết quả cho thấy chương trình học phù hợp với giáo trình, nhưng bài thi CSAT không đo hết những kỹ năng được đề cập đến trong chương trình học. Do đó, bài thi có tác động tiêu cực tới việc học và dạy tiếng Anh ở bậc học này tại Hàn Quốc. Đối tượng nghiên cứu cũng có thái độ tiêu cực đối với bài thi.

Stecher, Chun và Barron (2004) xem xét ảnh hưởng của đổi mới trong đánh giá kỹ năng viết tới việc dạy kỹ năng này tại bang Washington, Mỹ. Số liệu được thu thập qua khảo sát hiệu trưởng và giáo viên tại bang Washington. Một trong những mục đích chính của nghiên cứu là tìm ra ảnh hưởng của những đổi mới giáo dục đối với các hoạt động dạy và học. Nghiên cứu cho thấy việc dạy kỹ năng viết chịu ảnh hưởng nhiều từ bài thi và chính sách đánh giá mới. Giáo viên cho biết họ đã thay đổi nội dung bài học để phản ánh đúng bài thi. Phương pháp dạy cũng thay đổi: giáo viên thường xuyên giao nhiều bài tập để chuẩn bị cho kỳ thi. Theo các giáo viên tham gia phỏng vấn, việc thay thế, bổ sung các bài thi trắc nghiệm bằng các bài thi mang tính tự luận đánh giá năng lực đã làm tăng lượng viết của sinh viên tại trường lên đáng kể. Tuy nhiên, những nội dung, kỹ năng không thi cũng không được chú ý trong quá trình dạy và học.

Manjarres (2005) tìm hiểu ảnh hưởng của bài thi tiếng Anh tới việc dạy tiếng Anh trong hệ thống giáo dục Colombia. Số liệu của nghiên cứu được thu thập thông qua quan sát sư phạm, phỏng vấn và các bài kiểm tra tiếng Anh trong lớp. Kết quả cho thấy bài thi nhìn chung tạo ra hiệu ứng tích cực cho việc dạy và học tiếng Anh tại Colombia. Nhà nghiên cứu cũng nhấn mạnh bản chất phức tạp của hiện tượng tác động dội ngược và đề xuất nên sử dụng phương pháp nghiên cứu định tính để tìm hiểu hiện tượng này.

Trong bối cảnh giáo dục của Tây Ban Nha, Pizarro (2010) nghiên cứu ảnh hưởng của bài thi tiếng Anh quan trọng (highstakes English test) đối với việc dạy tiếng Anh ở các trường cấp 3. Một trong những mục đích của nghiên cứu là tìm hiểu ảnh hưởng của bài thi tới chương trình, tài liệu, phương pháp, tình cảm và thái độ của giáo viên. Số liệu thu thập qua bảng câu hỏi điều tra. Nghiên cứu tìm ra rằng bài thi có ảnh hưởng tới nhiều khía cạnh của chương trình học. Đặc biệt là tài liệu và phương pháp dạy bị ảnh hưởng theo hướng tiêu cực, chủ yếu tập trung chuẩn bị cho thí sinh tham dự kỳ thi. Giáo viên dành phần lớn thời gian trong lớp luyện tập các kỹ năng được thi và hầu như 
không dạy những kỹ năng, kiến thức không được thi. Do đó, bài thi làm khả năng giao tiếp của học sinh bị giảm đi rất nhiều do trong bài thi không có phần nghe và nói.

Nghiên cứu của Wang (2010) được tiến hành tại Trung Quốc. Nghiên cứu đánh giá tác động của bài thi tiếng Anh đại học (College English test - CET) đối với niềm tin và hoạt động thực hành của giáo viên. Nghiên cứu cũng xem xét những yếu tố phức tạp liên quan đến phương pháp, xã hội, cá nhân ảnh hưởng tới giáo viên. Kết quả của nghiên cứu cho thấy bài thi CET kết hợp với những yếu tố liên quan đến giáo viên tạo ra các tác động dội ngược mạnh mẽ của bài thi. Wang chỉ ra rằng do bản chất phức tạp của hiện tượng tác động dội ngược, thay đổi trong chính sách giáo dục và KTĐG không đủ để thay đổi hành vi và phương pháp của người giáo viên. Theo Wang, có 1 yếu tố chủ quan và 4 yếu tố khách quan ảnh hưởng đến hoạt động chuyên môn của giáo viên: hỗ trợ, phương pháp sư phạm, kinh nghiệm giảng dạy, trình độ ngôn ngữ và nhu cầu phát triển chuyên môn.

Trên đây là những nghiên cứu liên quan đến tác động của bài thi tiếng Anh với hoạt động dạy tại nhiều cơ sở giáo dục trên thế giới. Các nghiên cứu đều cho thấy một bài thi được đưa ra sẽ có ảnh hưởng dội ngược mạnh mẽ trở lại quá trình giảng dạy. Trong bối cảnh Việt Nam nói chung và ĐHNN-ĐHQGHN nói riêng, việc bước đầu đánh giá những ảnh hưởng của bài thi VSTEP khi mới được đưa vào làm thước đo chuẩn đầu ra ngoại ngữ của sinh viên là việc hết sức cần thiết, có ý nghĩa quan trọng trong việc đảm bảo chất lượng của hoạt động đào tạo.

\section{Một số tác động tích cực của bài thi đánh} giá năng lực tiếng Anh theo chuẩn đầu ra đối với việc dạy tiếng Anh tại ĐHNN - ĐHQGHN

Từ bài thi VSTEP đầu tiên vào ngày 16/5/2015, trải qua gần 2 năm được đưa vào sử dụng, đã xấp xỉ 2000 sinh viên thuộc ĐHNN - ĐHQGHN đăng ký dự thi bài thi này để xác định chuẩn đầu ra nhằm xét tốt nghiệp. Để đánh giá tác động của VSTEP đối với hoạt động giảng dạy tiếng Anh trong ĐHNN ĐHQGHN, chúng tôi đã tiến hành phỏng vấn sâu với 10 giảng viên thuộc Khoa Sư phạm tiếng Anh của ĐHNN - ĐHQGHN. Tất cả các giảng viên tham gia phỏng vấn đều có trình độ Thạc sĩ và kinh nghiệm giảng dạy từ 5 năm trở lên. Với số năm kinh nghiệm như vậy, giảng viên có những trải nghiệm với công việc giảng dạy từ trước khi VSTEP trở thành công cụ đo chính thức năng lực tiếng của người học, qua đó có thể có những nhận xét so sánh về hoạt động giảng dạy của họ trước và sau khi VSTEP được sử dụng.

Theo mô hình của Bailey (1996), các câu hỏi phỏng vấn tập trung vào khai thác các thông tin liên quan đến: (i) Quá trình xây dựng chương trình và thiết kế tài liệu, (ii) Hoạt động giảng dạy và kiểm tra đánh giá, (iii) Hoạt động nghiên cứu phục vụ giảng dạy, (iv) Ý kiến của giảng viên về hoạt động quản lý đào tạo. Các câu hỏi phỏng vấn được tiến hành theo hướng bán cấu trúc (semi-structured) để nhà nghiên cứu có thể linh động đặt các câu hỏi khai thác thêm dựa trên thông tin mà người tham gia đưa ra.

Theo đánh giá của toàn bộ 10 giáo viên tham gia phỏng vấn, bài thi nói chung đã tạo ra những thay đổi rất tích cực tới những người tham gia quá trình dạy và tổ chức dạy học, gồm có người xây dựng chương trình và viết tài liệu (curriculum designer, material writer), giáo viên (teachers), các nhà nghiên cứu (researchers) (theo mô hình của Bailey (1996)) và thậm chí là cả những nhà quản lý đào tạo. Kết quả trên trùng với phát hiện của Manjarres (2005).

\subsection{Xây dụng chưong trình và thiết kế tài liệu}

Theo những người được phỏng vấn, trước đây việc xây dựng chương trình thực hành 
tiếng còn mang nhiều tính chất cảm tính. Giữa các học kỳ không có sự phân định rõ về năng lực cần phát triển. Việc thiết kế chương trình chủ yếu dựa trên một số bộ giáo trình nước ngoài với những miêu tả trình độ sơ sài như Pre-intermediate (tiền trung cấp), Intermediate (trung cấp) và Advanced (cao cấp). Bản thân những người xây dựng chương trình và giảng viên cũng không hiểu rõ sự khác nhau giữa các bậc trình độ này, mà chỉ bằng kinh nghiệm cá nhân để phân biệt. Không hiếm gặp chuyện tiểu kỹ năng này đã được dạy ở kỳ trước nhưng được dạy lại ở kỳ sau hoặc tài liệu kỳ sau dễ hơn kỳ trước mặc dù về lý thuyết càng về các kỳ sau trình độ sinh viên đã càng được nâng cao.

Bài thi VSTEP ra đời trong bối cảnh chung việc áp dụng kiểm tra đánh giá theo hướng chuẩn đầu ra được tiến hành đồng bộ trong toàn bộ chương trình đào tạo của cả trường. Chính bài thi và việc đánh giá năng lực theo chuẩn đầu ra này là kim chỉ nam để Khoa Sư phạm tiếng Anh xây dựng lộ trình giảng dạy thực hành tiếng cho các kỳ học một cách logic và khoa học. Ví dụ, Khoa Sư phạm tiếng Anh đã đề ra một khung phát triển năng lực cho sinh viên chuyên Anh chương trình đào tạo chuẩn như sau:

\begin{tabular}{|c|c|c|}
\hline Học kỳ & CEFR & KNLNN \\
\hline Học kỳ 1 & B1+ & Bậc 3-Cao \\
\hline Học kỳ 2 & B2 & Bậc 4 \\
\hline Học kỳ 3 & B2+ & Bậc $4-$ Cao \\
\hline Học kỳ 4 & C1 & Bậc 5 \\
\hline
\end{tabular}

Như vậy, mỗi học kỳ đều có mục tiêu cụ thể, rõ ràng, được thông báo rộng rãi đến cả sinh viên và giáo viên để hướng tới. Thêm vào đó, với mỗi bậc năng lực đều được miêu tả cụ thể để tránh tình trạng, học kỳ sau dạy/phát triển lại những năng lực đã được phát triển tại học kỳ trước hoặc học kỳ sau, tài liệu dễ hơn tài liệu của kỳ trước. Ví dụ:
Năng lực nói tương tác ở bậc 3 được miêu tả: "Có thể sử dụng ngôn ngữ đơn giản để giải quyết hầu hết các tình huống có thể phát sinh trong khi đi du lịch. Có thể bắt đầu một cuộc hội thoại về những chủ để quen thuộc mà không cần chuẩn bị trước, thể hiện những quan điểm cá nhân và trao đổi thông tin về những chủ đề quen thuộc về cuộc sống thường ngày. Có thể giao tiếp tương đối tự tin về những vấn đề quen thuộc và không quen thuộc liên quan tới lĩnh vực chuyên môn hoặc quan tâm của mình. Có thể trao đổi, kiểm tra và xác nhận thông tin, xử lý các tình huống ít gặp và giải quyết vấn đề. Có thể bày tỏ suy nghĩ về những chủ đề văn hóa, có tính trừu tượng như phim ảnh, âm nhạc."

Năng lực bậc 4 lại được miêu tả cụ thể và nâng cao hơn: "Có thể giao tiếp khá lưu loát, tự nhiên, duy trì quan hệ với người bản ngữ mà không làm khó cho cả hai bên. Có thể giải trình ý quan trọng thông qua kinh nghiệm cá nhân, lý giải và duy trì quan điểm một cách rõ ràng với những lập luận và minh chứng liên quan. Có thể sử dụng ngôn ngữ thành thạo, chính xác và hiệu quả về các chủ đề chung, các chủ đề về giải trí, nghề nghiệp và học tập, tạo ra mối liên kết giữa các ý một cách rõ ràng. Có thể giao tiếp một cách tự nhiên, sử dụng tốt cấu trúc ngữ pháp và không gặp khó khăn khi diễn đạt phù hợp với hoàn cảnh."

Các giảng viên Khoa Sư phạm tiếng Anh cũng cho biết thêm: chương trình đã được cụ thể hóa đến từng những năng lực và tiểu kỹ năng cần phát triển cho mỗi học kỳ. Ngoài ra, ứng với mỗi bậc năng lực, còn có tài liệu liệt kê về các hiện tượng ngữ pháp, chủ đề, từ vựng, chức năng ngôn ngữ cần đạt được và những lỗi sai hay mắc phải ở từng bậc. Hệ thống tài liệu này được gọi là Learners' Outcome và Profile, đã giúp cụ thể hóa, lượng hóa những kiến thức, kỹ năng mà sinh viên cần đạt được trong từng học kỳ. Qua đó, sinh viên xây dựng được lộ trình phù hợp với năng lực của mình trên con đường đạt được trình độ 
bậc 5 hay $\mathrm{C} 1$ của bài thi VSTEP. Người thiết kế tài liệu bổ trợ cũng có cơ sở rõ ràng về ngữ pháp, từ vựng để thiết kế phù hợp với sinh viên từng học kỳ.

Những nhận định tích cực của nhóm giáo viên Khoa Sư phạm tiếng Anh dường như đi ngược lại với các nghiên cứu của Shohamy (1992), Hwang (2003) và Pizzaro (2010). Các nghiên cứu này đều cho rằng việc áp dụng bài thi chuẩn hóa đã tạo áp lực lớn cho giáo viên; giáo viên không sử dụng tài liệu giảng dạy mới nữa mà bắt đầu ôn tập lại tài liệu cũ; giáo trình được thay thế bởi các tờ bài tập giống như bài thi của những năm trước. Điều này vô tình làm méo mó mục tiêu và chương trình đào tạo, khiến lớp học phát triển năng lực tiếng thành lớp học luyện thi. Tuy nhiên, VSTEP đã tạo ra một hiệu ứng ngược lại khi thúc đẩy quá trình chuẩn hóa chương trình và tài liệu giảng dạy theo hướng có cơ sở khoa học về năng lực ngôn ngữ phù hợp với từng bậc năng lực.

Các nghiên cứu đi trước cũng đã chỉ ra một trong những yếu tố đảm bảo tính tích cực của bài thi là phải đảm bảo tính điển hình về nội dung và kỹ năng được kiểm tra. Người dự thi cần phải được học những kiến thức, kỹ năng xuất hiện trong bài thi. Ngược lại, nếu người dạy chỉ chú trọng dạy những kiến thức, kỹ năng phục vụ bài thi thì trải nghiệm giáo dục của người học sẽ bị hạn chế, dẫn đến lối học gạo thực dụng - cực kỳ nguy hiểm đối với những môn học mang tính thực hành cao như ngôn ngữ. Nghiên cứu của Hwang (2003) cho thấy bài thi CSAT đã có ảnh hưởng tiêu cực tới việc dạy và học tiếng Anh tại Hàn Quốc do bài thi không đo hết những kỹ năng được đề cập đến trong chương trình học. Người học thường bỏ qua những kỹ năng có trong giáo trình nhưng không có trong bài thi.

Ngược lại, nhóm giảng viên Khoa Sư phạm tiếng Anh tham gia phỏng vấn cho biết bài thi phản ánh khá tốt chương trình học và chương trình học cũng được điều chỉnh để cân bằng cả yếu tố phát triển năng lực tiếng và bổ sung kỹ thuật làm các bài thi chuẩn hóa: chương trình thực hành tiếng của Khoa Sư phạm tiếng Anh được chia thành 3 nhánh: Nhánh $\mathrm{A}$ - phát triển ngôn ngữ xã hội (ngôn ngữ được sử dụng trong ngữ cảnh đời thường), Nhánh $\mathrm{B}$ - phát triển ngôn ngữ học thuật (ngôn ngữ được sử dụng trong ngữ cảnh hàn lâm để phục vụ hoạt động học tập, nghiên cứu) và Nhánh $\mathrm{C}$ - phát triển kỹ năng làm bài thi. Đặc biệt, nhánh $\mathrm{C}$ không chỉ là luyện thi VSTEP mà tập trung phát triển toàn diện những kỹ năng làm bài thi nói chung. Ví dụ: kỹ năng nghe lấy ý chính, nghe lấy thông tin chi tiết, v.v. Với các kỹ năng được trang bị ở nhánh $\mathrm{C}$, sinh viên có thể tham gia làm bài ở bất cứ kỳ thi nào, chứ không chỉ VSTEP.

\subsection{Phương pháp giảng dạy và KTÐG}

8/10 giảng viên tham gia phỏng vấn cho biết về phương pháp giảng dạy, giáo viên ngày càng ít sử dụng phương pháp truyền thống mà chuyển sang nhiều phương pháp tích cực như dạy học theo nhóm, dạy học tích hợp, dạy học theo dự án. Tuy nhiên, có 2 giảng viên bổ sung thêm rằng đến gần kỳ thi những hình thức dạy học tích cực thường bị bỏ lại, giáo viên dành nhiều thời gian tập trung cho việc làm và chữa bài thi mẫu. Có một phát hiện tương đối thú vị rằng "chính sinh viên là người yêu cầu giảng viên giảm các bài tập giao tiếp để tập trung luyện và làm đề".

Về kiểm tra đánh giá, toàn bộ nhóm giảng viên tham gia phỏng vấn cho biết đã có những thay đổi lớn. Họ lý giải: do chương trình đã đặt ra những chuẩn cần đạt được cho mỗi học kỳ, việc kiểm tra đánh giá cũng đã thay đổi theo hướng phát triển năng lực được miêu tả cho mỗi bậc và cụ thể hóa hơn so với trước đây. Hình thức kiểm tra đánh giá trong quá trình (continuous assessment) được sử dụng thường xuyên hơn. Nhiều công cụ đánh giá phong phú, kết hợp đánh giá theo năng lực, 
chuẩn đầu ra và cá nhân hóa. Ở mỗi học kỳ, sinh viên phải thực hiện một số bài tập/dự án cá nhân và theo nhóm. Mỗi bài tập/dự án được thiết kế để đánh giá một số năng lực theo mục tiêu của từng môn học, ứng với bậc năng lực ngôn ngữ cần đạt được của kỳ đó. Dưới đây là ví dụ về mục tiêu năng lực được đánh giá của các bài tập lớn môn Tiếng Anh 3A.

\begin{tabular}{|c|c|}
\hline Bài tập lớn & $\begin{array}{c}\text { Năng lực được đánh giá (mã } \\
\text { hóa theo mục tiêu môn học } \\
\text { ghi trong đề cương môn học)* }\end{array}$ \\
\hline \multirow{3}{*}{ Tập bài viết } & $1.1,1.2$ \\
& $2.1,2.2$ \\
& $3.1,3.3,3.4,3.5$ \\
Thuyết trình & 4.2 \\
cá nhân & $1.1,1.2,1.3$ \\
& $2.1,2.2$ \\
\hline Luyện âm & $3.1,3.2,3.3,3.4$ \\
theo cặp & $4.1,4.2,4.3$ \\
\hline
\end{tabular}

*Ghi chú: Mục tiêu môn học cu thể xin xem trong Phu luc

Với các bài kiểm tra giữa kỳ và cuối kỳ, nếu như trước đây là sự tập hợp từ một số nguồn có độ khó tương tự với tài liệu dạy thì hiện nay đã có thiết kế mô tả kỹ thuật và ma trận cho từng bài kiểm tra dựa trên miêu tả năng lực của từng học kỳ. Mô tả kỹ thuật bao gồm: Mô tả sơ lược của bài thi, Mô tả chi tiết về ngữ liệu đầu vào, Mô tả chi tiết về yêu cầu cho các câu hỏi. Mô tả này sẽ là khung yêu cầu để tất cả các bài kiểm tra phải tuân theo nhằm đảm bảo việc thiết kế qua các năm, bởi những người khác nhau được thống nhất về nội dung, hình thức và mức độ khó. Mô tả được thiết kế phù hợp với mục tiêu môn học và nội dung bài học và được đưa vào đề cương môn học đảm bảo sinh viên và giáo viên đều viết trọng tâm của môn học, giúp việc dạy-học tập trung vào kiến thức, kỹ năng đầu ra mong đợi.

\subsection{Nghiên cúu}

Theo đánh giá chung của 9/10 người tham gia phỏng vấn, việc áp dụng bài thi chuẩn đầu ra VSTEP cũng tạo ra những thay đổi tích cực cho hoạt động nghiên cứu trong giáo dục ngoại ngữ của ĐHNN - ĐHQGHN. Bài thi đã mở ra một hướng nghiên cứu mới về kiểm tra đánh giá - một hướng còn mới mẻ ở Việt Nam. Hướng nghiên cứu này có thể coi là duy nhất ở Việt Nam và đang tiệm cận dần đến xu hướng của thế giới. Có thể nói ĐHNN ĐHQGHN là nơi đầu tiên có một hệ thống các nhà nghiên cứu theo sát từng bài thi từ khâu biên soạn đề, tổ chức thi, làm nghiên cứu xác trị và từ đó cải thiện dần bài thi. Việc nghiên cứu tính giá trị, độ tin cậy của bài thi một cách thường xuyên và bài bản đã góp phần nâng cao năng lực khảo thí ngoại ngữ của quốc gia, từng bước đưa bài thi VSTEP - bài thi chuẩn hóa đầu tiên của Việt Nam trở thành một bài thi được công nhận ở khu vực và thế giới. Người còn lại trong nhóm gia phỏng vấn cho biết giảng viên chưa được trang bị đủ kiến thức để có thể độc lập thực hiện những nghiên cứu chuyên sâu về kiểm tra đánh giá.

\subsection{Quản lý đào tạo}

Một số những câu hỏi phỏng vấn được đưa cho nhóm giảng viên tập trung xoay quanh ý kiến của họ về những hoạt động quản lý đào tạo của nhà trường khi sử dụng VSTEP làm thước đo chuẩn đầu ra ngoại ngữ. Hầu hết người tham gia phỏng vấn không nắm được thông tin này, chỉ có 2 giảng viên ở chức vụ quản lý biết đôi chút thông tin.

Trên thực tế, trong bối cảnh thị trường lao động ngày càng khắc nghiệt, hàng loạt cử nhân ngoại ngữ còn chưa tìm được việc làm, bài thi VSTEP như một lời cam kết về chất lượng đào tạo của ĐHNN - ĐHQGHN với xã hội. Đạt được bậc 5/6 (Khung năng lực ngoại ngữ 6 bậc dành cho Việt Nam) hay C1 (Khung tham chiếu châu Âu) là một thử thách lớn với bất cứ sinh viên chuyên ngữ nào. Nhưng khi đặt ra rào cho chính mình vượt qua, ĐHNN - ĐHQGHN cũng ngầm khẳng định rằng sản phẩm đào tạo của trường chắc chắn đáp ứng 
được yêu cầu khắt khe của bất cứ nhà tuyển dụng nào. Việc rà soát tỉ lệ đạt chuẩn đầu ra qua bài thi VSTEP định kỳ 4 lần/năm trước mỗi kỳ xét tốt nghiệp cũng giúp nhà trường có những biện pháp hỗ trợ kịp thời về mặt đào tạo cho sinh viên và điều chỉnh chương trình phù hợp, nhằm ngày càng hoàn thiện chương trình đào tạo của mình.

\section{Một số tác động tiêu cực và đề xuất}

Bên cạnh rất nhiều tác động tích cực của bài thi VSTEP đối với công tác đào tạo và quản lý đào tạo học phần thực hành tiếng ngôn ngữ Anh, vẫn còn một số những tác động cần sự chú ý của các nhà quản lý. Một số người tham gia phỏng vấn cho rằng: chuẩn đầu ra bậc $5 / 6$ (Khung năng lực ngoại ngữ 6 bậc dành cho Việt Nam) hay C1 (Khung tham chiếu châu Âu) là quá khó và gây nhiều căng thẳng cho sinh viên và cũng tạo nhiều áp lực cho giáo viên. Một số giáo viên đã tự động không tuân theo đề cương và lịch trình giảng dạy, dành nhiều thời gian luyện đề VSTEP. Điều này là cực kỳ nguy hiểm vì nó sẽ làm giảm trải nghiệm giáo dục của sinh viên, tập trung quá nhiều vào nội dung và kỹ năng được kiểm tra mà coi nhẹ những kiến thức và kỹ năng không được kiểm tra. Trong trường hợp này, bài thi đã làm "méo mó chương trình học", người học thường dùng thời gian để luyện tập các kỹ năng làm bài thi mà không tập trung thực sự vào việc học ngôn ngữ. Một số giảng viên cảm thấy không hài lòng khi chương trình thực hành tiếng trước năm 2014 được dạy trong 4 năm, nay giảm xuống còn 2 năm, hơn nữa lại dành hẳn học phần $3 \mathrm{C}$ và $4 \mathrm{C}$ để luyện kỹ thuật làm bài thi. Theo họ, do chương trình bị nén và thiên nhiều về luyện thi, không đủ thời lượng để giảng viên triển khai những bài tập phát triển năng lực ngôn ngữ.

Các nghiên cứu trên thế giới đã đề xuất nhiều giải pháp để biến những tác động tiêu cực thành tích cực. Hughes (1989) có đề xuất: (1) Giới thiệu rộng rãi định dạng bài thi tới người học và người dạy; (2) Hỗ trợ giáo viên khi cần thiết. Như vậy, trong bối cảnh tại ĐHNN - ĐHQGHN, hỗ trợ giáo viên ở đây có thể được hiểu là cần giúp bản thân giáo viên hiểu được về bài thi VSTEP - một bài thi còn tương đối mới. Nhà trường nên mở các khóa tập huấn để giảng viên hiểu về định dạng bài thi VSTEP, về phương pháp giảng dạy nhằm hướng đến chuẩn đầu ra bậc $5(\mathrm{C} 1)$. Bản thân các tổ chuyên môn cần xác định rõ những vướng mắc, khó khăn trong quá trình triển khai giảng dạy các học phần thực hành tiếng, từ đó tổ chức những buổi trao đổi chuyên môn và đề xuất với nhà trường về mặt chính sách.

Bachman \& Palmer (1996) đề xuất để tác động của bài thi là tích cực "cần tạo điều kiện cho người học tham gia vào quá trình thiết kế và phát triển bài thi, cũng như cần thu thập thông tin về quan niệm của họ về bài thi và các phần của bài thi”. Như vậy, sau mỗi bài thi VSTEP, các nhà nghiên cứu của ĐHNN có thể tiến hành khảo sát nhỏ để lấy ý kiến của người dự thi nhằm đánh giá bài thi có phản ánh đúng năng lực của họ và có phù hợp với chương trình đào tạo mà họ đã được học không. Ngoài ra, các tác giả cũng gợi ý cần cung cấp báo cáo chi tiết về điểm số và đảm bảo rằng kết quả đáng tin cậy và công bằng với người dự thi và người sử dụng kết quả bài thi. Tại ĐHNN, Trung tâm khảo thí vẫn đang tiến hành nghiên cứu xác trị trên kết quả mỗi bài thi VSTEP. Tuy nhiên, những con số và phân tích cần được thông báo rộng rãi đến giảng viên toàn trường, từ đó đưa ra những khuyến nghị để điều chỉnh tài liệu, phương pháp giảng dạy của họ.

\section{Kết luận}

Qua gần 2 năm được sử dụng làm bài thi chính thức xác định chuẩn đầu ra năng lực tiếng Anh tại ĐHNN - ĐHQGHN, bài thi VSTEP đã bước đầu có những tác động tích cực tới quá trình dạy và quản lý đào tạo các học phần thực hành tiếng ngôn ngữ Anh của 
nhà trường. Chương trình đã được cụ thể hóa tới từng những năng lực và tiểu kỹ năng cần phát triển cho mỗi học kỳ. Việc phát triển tài liệu bổ trợ đã có những căn cứ rõ ràng về đặc điểm ngôn ngữ cho từng bậc năng lực. Về phương pháp giảng dạy, giáo viên ngày càng ít sử dụng phương pháp truyền thống mà chuyển sang nhiều phương pháp tích cực, hiện đại. Kiểm tra đánh giá đã được làm bài bản, hệ thống hơn với các bảng mô tả kỹ thuật cho các bài thi định kỳ và các mô tả năng lực cần hướng tới cho các bài tập lớn. Bài thi cũng mở ra một hướng nghiên cứu mới về kiểm tra đánh giá - một hướng còn mới mẻ ở Việt Nam. Về quản lý đào tạo, bài thi là lời cam kêt về chất lượng đào tạo của nhà trường với xã hội; bài thi cũng là công cụ giúp nhà trường thường xuyên rà soát chất lượng đào tạo và có những thay đổi phù hợp.

Theo khảo sát ban đầu, có một số tác động không như mong đợi là một số giáo viên đã không tuân theo lịch trình giảng dạy mà dành nhiều thời gian cho luyện đề VSTEP, làm giảm trải nghiệm học tập của sinh viên, méo mó mục tiêu đào tạo; một số giáo viên cũng lo ngại về việc thời lượng học thực hành tiếng bị nén lại, không đủ để triển khai nhiều hoạt động học tập. Đề xuất đưa ra là cần giới thiệu rộng rãi định dạng bài thi và hỗ trợ giáo viên về phương pháp giảng dạy sao cho vừa phát triển năng lực ngôn ngữ của người học và vừa giúp người học chuẩn bị tốt cho kỳ thi VSTEP. Những người thiết kế bài thi cần lưu ý những đánh giá của người tham gia thi về bài thi. Kết quả bài thi và những phân tích cũng cần được thông tin rộng rãi cho giảng viên và các bên liên quan để có những điều chỉnh phù hợp về mặt nội dung và phương pháp giảng dạy.

Do số lượng người tham gia phỏng vấn không nhiều, nên những nhận định trên đây chỉ mang tính gợi mở về ảnh hưởng của bài thi VSTEP đối với hoạt động dạy tiếng Anh tại ĐHNN - ĐHQGHN. Trong thời gian tới, tác giả sẽ tiến hành nghiên cứu với số mẫu lớn hơn và mở rộng hơn tới cả tác động của bài thi tới hoạt động học tập của sinh viên.

\section{Tài liệu tham khảo}

Alderson, C., \& Wall, D. (1993). Does washback exist? Applied Linguistics, 14(2), 115-129.

Cheng, Watanabe, \& Curtis (2004). Washback in language testing. London: Lawrence Erlbaum Associates.

Cunningsworth, A. (1995). Choosing your coursebook. Oxford: Heinemann Educational Books.

Damankesh, M. \& Babaii, E. (2014). The washback effect of Iranian high school final examinatins on students' test-taking and test-prepration strategies. Studies in Educational Evaluation, 45 (2015), 62-69.

Davies, A. (1985). Language testing symposium: $A$ psycholinguistic approach. Oxford: Oxford University Press.

Estaji, M. \& Tajeddin, Z. (2012). The learner factor in washback context: An emperical study investigating the washback of the IELTS Academic Writing test. Language Testing in Asia, 2(1), 5-25.

Fredericksen, R. \& Collins, A. (1989). A system approach to educational testing. Educational Researcher 18 (27), 27-32

Gosa, C. M. C. (2004). Investigating washback: A case study using students' diaries. Unpublished $\mathrm{PhD}$ dissertation. Lancaster: Lancaster University.

Heyneman, S. P., \& Ranson, A, W. (1990). Using examinations and testing to improveeducational quality. Educational Policy, 4. 177-192.

Hughes, A. (1993). Backwash and TOEFL 2000. Unpublished manuscript. UK: University of Reading.

Hwang, H. J. (2003). The impact of high-stakes exams on teachers and students: A washback study of the university entrance exam at the secondary school level in South Korea. Master of Arts, Digital Dissertations. Quebec: McGill University.

Kellaghan T. \& Greaney, V. (1992). Using examinations to improve education: A study of fourteen African countries. Washington DC: The World Bank.

Mahmoudi, L. (2014). The washback effects of Iranian national university entrance exam on pre-university English teaching and learning. $\mathrm{PhD}$ thesis, Malaya University, Kuala lumpur, Malaysia.

Manjarres, N. B. (2005). Washback of the foreign language test of the state examinations in 
Colombia: A case study. Arizona Working Papers in SLAT, 12, 1-19.

Pizarro , M.A. (2010). Exploring the washback effects of a high-stakes English test on the teaching of English in Spanish upper secondary schools. Revista Alicantina de Estudios Ingleses, 23, 149-170.

Popham, W.J. (1983). Measurement as an instructional catalyst. In R.B Ekstrom (Ed.). Newdirections for testing and measurement, Measurement, Technology, and Individuality Education (pp.1930). San Francisco, Jossey-Bass.

Prodromou, L. (1995). The backwash effect: From testing to teaching. ELT Journal, 49(1), 13-25.

Shohamy, E. (1992). Beyond proficiency testing: A diagnostic feedback testing model for assessing foreign language learning. Modern Language Journal, 76 (4), 513-521.

Shohamy, E. (2001). The power of tests. London: Longman/ Pearson

Shohamy, E., Donista-Schmidt, S. \& Ferman, I. (1996). Test Impact revisited, washback effect over time. Language Testing 13(3), 298-317.

Stecher, B., Chun, T. \& Barron, S. (2004). Effects of assessment-driven reform on the teaching of writing in Washington state. In Cheng, L. and Y. Watanabe (Eds.), Washback in language testing: Research contexts and methods, (pp.53-69). Lawrence Earlbaum.
Tsagari, D. (2011). Washback of a high-stakes English exam on teachers' perceptions and practices. Selected papers from the 19th ISTAL.

Vernon, P. E. (1956). The measurement of abilities (the 2nd ed). London: University of London Press.

Watanabe, Y. (1990). External variables affecting language learning strategies of Japanese EFL learners: Effects of entrance examination, year spent at college/university and staying overseas. Unpublished MA thesis, Lancaster University.

Watanabe, Y. (1996). Does grammar translation come from the entrance examination? Preliminary findings from classroom-based research. Language Testing, 13(3), 318-333.

Wall, D. (1997). Impact and washback in language testing, In C. C. \& D. Corson(Eds.), Encyclopaedia of language and education, Language Testing and Assessment 7.

Wiseman, S. (Ed.). (1961). Examinations and English education. Manchester: Manchester University Press.

Wall, D. \& Alderson, J.C. (1993). Examining washback: the Sri Lanka impact study. Language Testing, 10(1), 41-69.

\title{
EXPLORING THE WASHBACK EFFECTS OF VSTEP ON THE TEACHING OF ENGLISH AT ULIS - VNU
}

\author{
Nguyen Thuy Lan \\ Academic Affairs Department, VNU University of Languages and International Studies, \\ Pham Van Dong, Cau Giay, Hanoi, Vietnam
}

\begin{abstract}
The assessment of learning outcomes and the process of teaching are closely related. When implemented at the end stage of a training process, the assessment can provide information about learners' completion of training objectives; however, the assessment can also have significant washback effects on the training process itself. After nearly 2 years of being used as the official English language standardized exam to determine the English competency as a learning outcome at ULIS-VNU, VSTEP initially has major impact on the teaching and management of English skill development subjects. This article aims at exploring some positive effects and some others that need ULIS managers' attention, followed by a number of proposals to improve the effectiveness of the test in regulating the training process.
\end{abstract}

Keywords: washback effect, English competency assessment, learning outcome 


\section{PHỤ LỤC 1 \\ MỤC TIÊU KHÓA HỌC CHI TIẾT (ENGLISH 3A)}

\section{In terms of linguistic competence}

Students should be able to use a sufficient range of language to give clear descriptions, express viewpoints and develop arguments without much conspicuous searching for words. More specifically, they can:

\subsection{Lexical competence}

\section{Range}

1.1.a.1 activate a broad vocabulary with a large degree of independence in a variety of topics including Education, Crimes and Punishment, Business and Trade, Entertainment, Money, Plants and Animals.

1.1.a.2 use a range of complex vocabulary to express abstract ideas as well as topical subjects and correct their own mistakes in the process

\section{Control}

1.1.b.1 understand correspondences related to their personal interests, read articles and short stories with occasional use of dictionary

1.1.b.2 distinguish lexical elements in the perception or production of messages

1.1.b.3 convey the meaning of word, including connotation, relation of words to general context, synonyms/antonyms, hyponyms, collocations, etc.

\subsection{Grammatical competence}

1.2.1 use a system of principles governing the assembly of elements into meaningful organized sentences

1.2.2 recognize, understand and express meaning by means of well-formed sentences in accordance with the rules of morphology, syntax, grammatical semantics of sentences and phonology
1.2.3 maintain good grammatical control despite a few mistakes, which are subject to later self-correction

\subsection{Phonological competence}

1.3.1 recognize, perceive and produce distinct, meaningful sounds (phonemes), including consonants and vowels, assimilation of sounds, phonetic composition of words and sentences, including tones and intonations of a voice, rhythm, sentence stress and other characteristics carrying meaning.

1.3.2 understand in detail a range of common spoken discourses such as TV documentaries, interviews, talk shows, plays, films, announcements, instructions, telephone messages etc. even in a noisy environment and when they are spoken fast, provided they are in standard dialect.

1.3.3 keep up with animated discussions between native speakers.

\section{In terms of sociolinguistic competence}

Students should be able to express themselves confidently, clearly, and politely in a formal or informal register, appropriate to situations and persons concerned, including: Conversing about everyday topics, Discussing about controversial statements, Asking for help, Telling stories, Comparing things, Explaining viewpoints, and Reporting questions and answers. More specifically, they can:

\subsection{Register appropriacy}

2.1.1 Apply a range of linguistic markers related to the social situations listed above

2.1.2 Recognize the register differences caused by participant and situational variables (typical lexicon, grammar, phonology) and adapt the language use aligned with the level of formality (prescribed grammar, the use of academic, uncommon words, contractions, direct or indirect speech, etc.)

2.1.3 Appreciate register shifts but may need to confirm occasional details, especially if the accent is unfamiliar. 


\subsection{Stype appropriacy}

2.2.1 Use an assured, personal, natural style appropriate to readers/ listeners

\section{In terms of pragmatic competence}

Students should be able to coherently organize, structure and arrange messages (opinions on short stories, articles, talks, discussions and interviews); sequence them according to specific communicative designs (reports, for-and-against essays, letters, reviews); perform certain communicative functions (narrate stories, carry out interviews, summarize films/ plays), in oral and written forms. More specifically, they can:

\subsection{Flexibility}

3.1.1 reformulate ideas in different ways to ensure people understand exactly what they mean

3.1.2 adjust what they say and the means of expressing it to the situation and the recipient and adopt a level of formality appropriate to the circumstances

\subsection{Turn taking}

3.2.1 use stock phrases to gain time and keep the turn whilst formulating what to say

3.2.2 initiate, maintain and end discourse appropriately with effective turn-taking

3.2.3 intervene appropriately in discussion, exploiting appropriate language to do so

\subsection{Thematic Development}

3.3.1 give clear, well-developed, detailed descriptions on the subjects listed above, expanding and supporting their ideas

3.3.2 comprehend and produce meaningful messages which are sequenced according to interactional and transactional communicative design (question - answer, statement - agreement/disagreement, request/ offer/apology - acceptance/refusal, greeting response)

\subsection{Coherence}

3.4.1 use a variety of linking words efficiently to mark clearly the relationships between ideas

3.4.2 use a limited range of cohesive devices to link utterances into clear, coherent discourse

\subsection{Propositional-precision}

3.5.1 pass on detailed information reliably

\section{In terms of strategic competence}

Students should be able to plan, execute, evaluate and repair their oral and written communication. More specifically, they can:

\subsection{Reception strategies}

4.1.1 adapt the styles and speed of reading to unfamiliar texts for a range of different purposes

4.1.2 keep up with an animated conversation between native speakers even on familiar topics

\subsection{Production strategies}

4.2.1 plan what is to be written/spoken and the means to express it, considering the recipients

4.2.2 monitor their speech and writing to correct slips and mistakes that they make

4.2.3 organize and purposefully regulate a line of communicative verbal and non-verbal actions selected for the achievement of communicative goals in a certain context and in specific conditions especially if there is insufficiency in linguistic and socio-cultural knowledge

4.2.4 solve (unexpectedly occurred) communicative problems

\subsection{Interactive strategies}

4.3.1 develop a discussion by giving feedback, follow up what people say and relate the students' contribution to their partners' 


\section{PHỤ LỤC 2}

\section{CÁC CÂU HỎI PHỎNG VẤN}

1. Theo anh/chị, trước khi VSTEP được áp dụng (trước năm 2015), việc xây dựng chương trình được dựa trên những nguyên tắc nào?

2. Theo anh/chị, trước khi VSTEP được áp dụng (trước năm 2015), việc chọn giáo trình, thiết kế tài liệu được thực hiện như thế nào?

3. Theo anh/chị, từ năm 2015 trở về trước, các phương pháp giảng dạy chủ yếu mà anh chị sử dụng trong các giờ thực hành tiếng là gì?

4. Trước năm 2015, khi thiết kế các bài thi giữa kỳ, cuối kỳ, anh/chị làm như thế nào?

5. Trước năm 2015, anh/chị thường nghiên cứu, viết bài báo về những chủ đề gì? Các anh/chị thấy trong các hội thảo khoa học, các nghiên cứu thường tập trung vào vấn đề gì?
6. Từ khi VSTEP được sử dụng như một bài thi chuẩn hóa để xác định chuẩn đầu $\mathrm{ra}, \mathrm{anh} / \mathrm{ch}$ ị thấy có gì thay đổi trên các phương diện sau đây không? Nếu có, hãy chỉ rõ.

- Xây dựng chương trình

- Lựa chọn giáo trình

- Thiết kế tài liệu

- Phương pháp giảng dạy

- Kiểm tra đánh giá

- Nghiên cứu

7. Anh/chị có biết nhà trường (ĐHNN) có những biện pháp/hoạt động quản lý đào tạo nào mới liên quan đến bài thi VSTEP không? Nếu có, anh/chị có nhận xét gì về những biện pháp/hoạt động đó?

8. Anh/chị có đề xuất gì với nhà trường $(\mathrm{PHNN})$ liên quan đến bài thi VSTEP không? 